\title{
Generalized Mittag-Leffler Function Associated with Weyl Fractional Calculus Operators
}

\author{
Ahmad Faraj, ${ }^{1}$ Tariq Salim, ${ }^{1}$ Safaa Sadek, ${ }^{2}$ and Jamal Ismail ${ }^{2}$ \\ ${ }^{1}$ Department of Mathematics, Al-Azhar University-Gaza, P.O. Box 1277, Gaza, Palestine \\ ${ }^{2}$ Department of Mathematics, College of Girls Ain Shams University, Cairo, Egypt
}

Correspondence should be addressed to Tariq Salim; trsalim@yahoo.com

Received 8 January 2013; Revised 1 April 2013; Accepted 18 April 2013

Academic Editor: Josefa Linares-Perez

Copyright (c) 2013 Ahmad Faraj et al. This is an open access article distributed under the Creative Commons Attribution License, which permits unrestricted use, distribution, and reproduction in any medium, provided the original work is properly cited.

This paper is devoted to study further properties of generalized Mittag-Leffler function $E_{\alpha, \beta, p}^{\gamma, \delta, q}$ associated with Weyl fractional integral and differential operators. A new integral operator $\mathscr{E}_{\alpha, \beta, p, w, \infty}^{\gamma, \delta, q}$ depending on Weyl fractional integral operator and containing $E_{\alpha, \beta, p}^{\gamma, \delta, q}(z)$ in its kernel is defined and studied, namely, its boundedness. Also, composition of Weyl fractional integral and differential operators with the new operator $\mathscr{E}_{\alpha, \beta, p, w, \infty}^{\gamma, \delta, q}$ is established.

\section{Introduction}

In 1903, the Swedish mathematician Mittag-Leffler [1] introduced the function $E_{\alpha}(z)$ as

$$
E_{\alpha}(z)=\sum_{n=0}^{\infty} \frac{z^{n}}{\Gamma(\alpha n+1)},
$$

where $z \in \mathbb{C}$ and $\Gamma(s)$ is the gamma function; $\alpha \geq 0$.

During the last century and due to its involvement in the problems of physics, engineering, and applied sciences, many authors defined and studied in their research papers different generalizations of Mittag-Leffler type function, namely, $E_{\alpha, \beta}(z)$ introduced by Wiman [2], $E_{\alpha, \beta}^{\gamma}(z)$ stated by Prabhakar [3], $E_{\alpha, \beta}^{\gamma, q}(z)$ defined and studied by Shukla and Prajapati [4], and $E_{\alpha, \beta}^{\gamma, \delta}(z)$ investigated by Salim and Faraj [5].

Prabhakar studied some properties of generalized MittagLeffler type-function $E_{\alpha, \beta}^{\gamma}(z)$ and the fractional integral operator

$$
\left(\mathscr{E}_{\alpha, \beta, w, a^{+}}^{\gamma} \varphi\right)(x)=\int_{a}^{x}(x-t)^{\beta-1} E_{\alpha, \beta}^{\gamma}\left[w(x-t)^{\alpha}\right] \varphi(t) d t
$$

containing $E_{\alpha, \beta}^{\gamma}(z)$ in the kernel and applied the result obtained to prove the existence and uniqueness of the solution of corresponding integral equation of the first kind.
Moreover, Kilbas et al. [6] devoted themselves to further investigation of $E_{\alpha, \beta}^{\gamma}(z)$, and the integral operator defined in (2). They established integral representation, differentiation and integration properties of $E_{\alpha, \beta}^{\gamma}(z)$ and formulas of its Riemann-Liouville fractional integral and differential operators. For more results and conclusions, one can refer to the work of Srivastava and Tomovski [7].

Recently, Salim and Faraj [5] introduced a new generalization of Mittag-Leffler-type function as

$$
E_{\alpha, \beta, p}^{\gamma, \delta, q}(z)=\sum_{n=0}^{\infty} \frac{(\gamma)_{q n} z^{n}}{\Gamma(\alpha n+\beta)(\delta)_{p n}}
$$

where

$$
\begin{gathered}
z, \alpha, \beta, \gamma, \delta \in \mathbb{C} ; \min \{\operatorname{Re}(\alpha), \operatorname{Re}(\beta), \operatorname{Re}(\gamma), \operatorname{Re}(\delta)\}>0, \\
p, q>0 .
\end{gathered}
$$

Equation (3) is just a generalized formula of Mittag-Leffler function; its various properties including differentiation, Laplace, Beta, and Mellin transforms, and generalized hypergeometric series form and its relationship with other type of special functions were investigated and established. 
On the other hand Salim and Faraj in their research paper defined and studied an integral operator $\mathscr{E}_{\alpha, \beta, p, w, a^{+}}^{\gamma, \delta, q}$ as

$$
\left(\mathscr{E}_{\alpha, \beta, p, w, a^{+}}^{\gamma, \delta, q} \varphi\right)(x)=\int_{a}^{x}(x-t)^{\beta-1} E_{\alpha, \beta, p}^{\gamma, \delta, q}\left[w(x-t)^{\alpha}\right] \varphi(t) d t
$$

containing $E_{\alpha, \beta, p}^{\gamma, \delta, q}(z)$ in the kernel. Also, composition of Riemann-Liouville fractional integral and differential operators with the integral operator defined in (5) was established.

This paper is devoted for the study of further properties of the generalized Mittag-Leffler function $E_{\alpha, \beta, p}^{\gamma, \delta, q}(z)$ defined in (3) with another type of fractional calculus operators called Weyl fractional integral and differential operators written as:

$$
\begin{gathered}
\left(I_{-}^{\lambda} \varphi\right)(x)=\frac{1}{\Gamma(\lambda)} \int_{x}^{\infty}(t-x)^{\lambda-1} \varphi(t) d t \\
\left(D_{-}^{\lambda} \varphi\right)(x) \\
=(-1)^{m}\left(\frac{d}{d x}\right)^{m} \frac{1}{\Gamma(m-\lambda)} \int_{x}^{\infty}(t-x)^{m-\lambda-1} \varphi(t) d t
\end{gathered}
$$

The last definition can be written in the form

$$
\left(D_{-}^{\lambda} \varphi\right)(x)=(-1)^{m}\left(\frac{d}{d x}\right)^{m}\left(I_{-}^{m-\lambda} \varphi\right)(x) .
$$

Precisely, the authors investigate the basic properties of Weyl fractional integral and differential operator with generalized Mittag-Leffler function $E_{\alpha, \beta, p}^{\gamma, \delta, q}(z)$; moreover, a new integral operator depending on Weyl fractional integral operator and containing $E_{\alpha, \beta, p}^{\gamma, \delta, q}(z)$ in its kernel is established as

$$
\left(\mathscr{E}_{\alpha, \beta, p, w, \infty}^{\gamma, \delta, q} \varphi\right)(w)=\int_{x}^{\infty}(t-x)^{\beta-1} E_{\alpha, \beta, p}^{\gamma, \delta, q}\left[w(t-x)^{\alpha}\right] \varphi(t) d t
$$

The condition of boundedness of the integral operator (9) is discussed and stated in the space $L(a, \infty)$ of Lebesguemeasurable functions on $(a, \infty)$

$$
L(a, \infty)=\left\{g(x):\|g\|_{1}=\int_{a}^{\infty}|g(x)| d x<\infty\right\} .
$$

Also, composition of Weyl fractional integration and differentiation with the operator defined in (9) is established.

Throughout this paper, we need the following well-known facts and rules.

(i) Fubini’s theorem (Dirichlet formula) [8]

$$
\begin{gathered}
\int_{a}^{b} d x \int_{a}^{x} f(x, t) d t=\int_{a}^{b} d t \int_{t}^{b} f(x, t) d x, \\
\frac{d}{d x} \int_{a}^{x} h(x, t) d t=\left[\int_{a}^{x} \frac{\partial}{\partial x} h(x, t) d t\right]+h(x, x) .
\end{gathered}
$$

(ii) The Riemann-Liouville fractional integral [8]

$$
\begin{array}{r}
\left(I_{a^{+}}^{\lambda} \varphi\right)(x)=\frac{1}{\Gamma(\lambda)} \int_{a}^{x}(x-t)^{\lambda-1} \varphi(t) d t \\
(\alpha \in \mathbb{C}, \operatorname{Re}(\alpha)>0) .
\end{array}
$$

(iii) The Riemann-Liouville fractional derivative [8]

$$
\left(D_{a^{+}}^{\lambda} \varphi\right)(x)=\left(\frac{d}{d x}\right)^{n}\left(I_{a^{+}}^{n-\lambda} \varphi\right)(x) \quad n=[\operatorname{Re}(\alpha)]+1 .
$$

(iv) Beta transform (Sneddon [9])

$$
B\{f(z) ; a, b\}=\int_{0}^{1} z^{a-1}(1-z)^{b} f(z) d z
$$

where $\operatorname{Re}(a)>0, \operatorname{Re}(b)>0$.

(v) The Beta function is written as:

$$
\beta(\alpha, \beta)=\frac{\Gamma(\alpha) \Gamma(\beta)}{\Gamma(\alpha+\beta)}
$$

(vi) The difference property of the Gamma function is

$$
\Gamma(\alpha+1)=\alpha \Gamma(\alpha)
$$

\section{Further Properties of Weyl Fractional Integral Related to Mittag-Leffler Function}

In this section, we consider composition of Weyl fractional integral and derivative (6) and (7) with generalized MittagLeffler function $E_{\alpha, \beta, p}^{\gamma, \delta, q}(z)$ defined in (3).

Theorem 1. Let $\alpha, \beta, \gamma, \delta, \lambda, w \in \mathbb{C} ; \min \{\operatorname{Re}(\alpha), \operatorname{Re}(\beta), \operatorname{Re}(\gamma)$, $\operatorname{Re}(\delta), \operatorname{Re}(\lambda)\}>0$ and $p, q>0$, then

$$
I_{-}^{\lambda}\left[t^{-\lambda-\beta} E_{\alpha, \beta, p}^{\gamma, \delta, q}\left(w t^{-\alpha}\right)\right](x)=x^{-\beta} E_{\alpha, \beta+\lambda, p}^{\gamma, \delta, q}\left(w x^{-\alpha}\right) .
$$

Proof.

$$
\begin{aligned}
& I_{-}^{\lambda}\left[t^{-\lambda-\beta} E_{\alpha, \beta, p}^{\gamma, \delta, q}\left(w t^{-\alpha}\right)\right](x) \\
& =\frac{1}{\Gamma(\lambda)} \int_{x}^{\infty}(t-x)^{\lambda-1} t^{-\lambda-\beta} \\
& \quad \times \sum_{n=0}^{\infty} \frac{(\gamma)_{q n} w^{n} t^{-\alpha n}}{(\delta)_{p n} \Gamma(\alpha n+\beta)} d t \\
& =\frac{1}{\Gamma(\lambda)} \sum_{n=0}^{\infty} \frac{(\gamma)_{q n} w^{n}}{(\delta)_{p n} \Gamma(\alpha n+\beta)} \\
& \quad \times \int_{x}^{\infty}(t-x)^{\lambda-1} t^{-\lambda-\beta} t^{-\alpha} d t .
\end{aligned}
$$


Let $u=(t-x) / t$, then

$$
\begin{aligned}
& I_{-}^{\lambda}\left[t^{-\lambda-\beta} E_{\alpha, \beta, p}^{\gamma, \delta, q}\left(w t^{-\alpha}\right)\right](x) \\
& =\frac{1}{\Gamma(\lambda)} \sum_{n=0}^{\infty} \frac{(\gamma)_{q n} w^{n} x^{-\beta-\alpha n}}{(\delta)_{p n} \Gamma(\alpha n+\beta)} \\
& \times \int_{0}^{1} u^{\lambda-1}(1-u)^{\alpha n+\beta-1} d u \\
& =\frac{1}{\Gamma(\lambda)} \sum_{n=0}^{\infty} \frac{(\gamma)_{q n} w^{n} x^{-\beta-\alpha n}}{(\delta)_{p n} \Gamma(\alpha n+\beta)} B(\lambda, \alpha n+\beta) \\
& =x^{-\beta} E_{\alpha, \beta+\lambda, p}^{\gamma, \delta, q}\left(w x^{-\alpha}\right) .
\end{aligned}
$$

Theorem 2. Let $\alpha, \beta, \gamma, \delta, \lambda, w \in \mathbb{C} ; \min \{\operatorname{Re}(\alpha), \operatorname{Re}(\beta), \operatorname{Re}(\gamma)$, $\operatorname{Re}(\delta), \operatorname{Re}(\lambda)\}>0, \operatorname{Re}(\beta)>[\operatorname{Re}(\lambda)]+1$ and $p, q>0$, then

$$
D_{-}^{\lambda}\left[t^{\lambda-\beta} E_{\alpha, \beta, p}^{\gamma, \delta, q}\left(w t^{-\alpha}\right)\right](x)=x^{-\beta} E_{\alpha, \beta-\lambda, p}^{\gamma, \delta}\left(w x^{-\alpha}\right)
$$

Proof. Making use of (7), we get

$$
\begin{aligned}
D_{-}^{\lambda}\left[t^{\lambda-\beta} E_{\alpha, \beta, p}^{\gamma, \delta, q}\left(w t^{-\alpha}\right)\right](x) \\
=(-1)^{m}\left(\frac{d}{d x}\right)^{m} \frac{1}{\Gamma(m-\lambda)} \\
\quad \times \int_{x}^{\infty}(t-x)^{m-\lambda-1} t^{\lambda-\beta} \sum_{n=0}^{\infty} \frac{(\gamma)_{q n}\left(w t^{-\alpha}\right)^{n}}{(\delta)_{p n} \Gamma(\alpha n+\beta)} d t \\
=(-1)^{m}\left(\frac{d}{d x}\right)^{m} \frac{1}{\Gamma(m-\lambda)} \\
\quad \times \sum_{n=0}^{\infty} \frac{w^{n}(\gamma)_{q n}}{(\delta)_{p n} \Gamma(\alpha n+\beta)} \\
\quad \times \int_{x}^{\infty}(t-x)^{m-\lambda-1} t^{\lambda-\beta} t^{-\alpha n} d t .
\end{aligned}
$$

Let $u=(t-x) / t$, then

$$
\begin{aligned}
D_{-}^{\lambda} & {\left[t^{\lambda-\beta} E_{\alpha, \beta, p}^{\gamma, \delta, q}\left(w t^{-\alpha}\right)\right](x) } \\
= & (-1)^{m}\left(\frac{d}{d x}\right)^{m} \frac{1}{\Gamma(m-\lambda)} \\
& \quad \times \sum_{n=0}^{\infty} \frac{(\gamma)_{q n} w^{n} x^{m-\alpha n-\beta}}{(\delta)_{p n} \Gamma(\alpha n+\beta)} \int_{0}^{1} u^{m-\lambda-1}(1-u)^{\alpha n+\beta-m-1} d u \\
= & (-1)^{m}\left(\frac{d}{d x}\right)^{m} \frac{1}{\Gamma(m-\lambda)}
\end{aligned}
$$

$$
\begin{aligned}
& \times \sum_{n=0}^{\infty} \frac{(\gamma)_{q n} w^{n} x^{m-\alpha n-\beta}}{(\delta)_{p n} \Gamma(\alpha n+\beta)} B(m-\lambda, \alpha n+\beta-m) \\
= & (-1)^{m} \sum_{n=0}^{\infty} \frac{(\gamma)_{q n} w^{n} \Gamma(\alpha n+\beta-m)}{(\delta)_{p n} \Gamma(\alpha n+\beta) \Gamma(\alpha n+\beta-\lambda)} \cdot(m-\lambda n-\beta) \\
= & (-1)^{m} \sum_{n=0}^{\infty} \frac{(\gamma)_{q n} w^{n} \Gamma(\alpha n+\beta-m)}{(\delta)_{p n} \Gamma(\alpha n+\beta) \Gamma(\alpha n+\beta-\lambda)} \\
= & x^{-\beta} \sum_{n=0}^{\infty} \frac{(\gamma)_{q n}\left(w x^{-\alpha}\right)^{n}}{(\delta)_{p n} \Gamma(\alpha n+\beta-\lambda)} \\
= & x^{-\beta} E_{\alpha, \beta-\lambda, p}^{\gamma, \delta, q}\left(w x^{-\alpha}\right) .
\end{aligned}
$$

\section{Weyl Integral Operator with Generalized Mittag-Leffler Function in the Kernel}

Consider the Weyl integral operator defined in (9) containing $E_{\alpha, \beta, p}^{\gamma, \delta, q}(z)$ in the kernel. First of all, we prove that the operator $\mathscr{E}_{\alpha, \beta, p, w, \infty}^{\gamma, \delta, q}$ is bounded on $L(a, \infty)$.

Theorem 3. Let $\alpha, \beta, \gamma, \delta, \lambda, w \in \mathbb{C}$; with $\min \{\operatorname{Re}(\alpha), \operatorname{Re}(\beta)$, $\operatorname{Re}(\gamma), \operatorname{Re}(\delta), \operatorname{Re}(\lambda)\}>0$ and $p, q>0$, then the operator $\mathscr{E}_{\alpha, \beta, p, w, \infty}^{\gamma, \delta, q}$ is bounded on $L(a, \infty)$ and

$$
\left\|\mathscr{E}_{\alpha, \beta, p, w, \infty}^{\gamma, \delta, q} \varphi\right\|_{1} \leq \beta\|\varphi\|_{1}
$$

where

$$
\beta=(b-a)^{\operatorname{Re}(\beta)} \sum_{n=0}^{\infty} \frac{\left|(\gamma)_{q n}\right|\left|w(b-a)^{\alpha}\right|^{n}}{\left|(\delta)_{p n}\right||\Gamma(\alpha n+\beta)||\operatorname{Re}(\alpha) n+\operatorname{Re}(\beta)|} .
$$

Proof. Let $C_{n}$ denote the $n$th term of (25), then

$$
\begin{aligned}
\left|\frac{C_{n+1}}{C_{n}}\right|= & \left|\frac{(\gamma)_{q n+q}}{(\gamma)_{q n}}\right|\left|\frac{\Gamma(\alpha n+\beta)}{\Gamma(\alpha n+\beta+\alpha)}\right|\left|\frac{(\delta)_{p n}}{(\delta)_{p n+p}}\right| \\
& \times\left|\frac{\operatorname{Re}(\alpha) n+\operatorname{Re}(\beta)}{\operatorname{Re}(\alpha) n+\operatorname{Re}(\alpha)+\operatorname{Re}(\beta)}\right|\left|w(b-a)^{\operatorname{Re}(\alpha)}\right| \\
\approx & \frac{\left|w(b-a)^{\operatorname{Re}(\alpha)}\right|(q n)^{q}}{(|\alpha| n)^{\operatorname{Re}(\alpha)}(p n)^{p}} \text { as } n \longrightarrow \infty .
\end{aligned}
$$

Hence, $\left|C_{n+1} / C_{n}\right| \rightarrow 0$ as $n \rightarrow \infty$ and $q<p+\operatorname{Re}(\alpha)$ which means that the right-hand side of (25) is convergent and finite under the given condition. 
Now, according to (9), (10), and (11), we get

$$
\begin{aligned}
& \left\|\mathscr{E}_{\alpha, \beta, p, w, \infty}^{\gamma, \delta, q} \varphi\right\|_{1} \\
& \quad=\int_{a}^{\infty}\left|\int_{x}^{\infty}(t-x)^{\beta-1} E_{\alpha, \beta, p}^{\gamma, \delta, q}\left[w(t-x)^{\alpha}\right] \varphi(t) d t\right| d x \\
& \quad \leq \int_{a}^{\infty}\left[\int_{a}^{t}(t-x)^{\beta-1}\left|E_{\alpha, \beta, p}^{\gamma, \delta, q}\left[w(t-x)^{\alpha}\right]\right| d x\right]|\varphi(t)| d t \\
& \quad=\int_{a}^{\infty}\left[\int_{0}^{t-a} u^{\operatorname{Re}(\beta)-1}\left|E_{\alpha, \beta, p}^{\gamma, \delta, q}\left(w u^{\alpha}\right)\right| d u\right]|\varphi(t)| d t \\
& \quad \leq \int_{a}^{\infty}\left[\int_{0}^{b-a} u^{\operatorname{Re}(\beta)-1}\left|E_{\alpha, \beta, p}^{\gamma, \delta, q}\left(w u^{\alpha}\right)\right| d u\right]|\varphi(t)| d t .
\end{aligned}
$$

Let

$$
\int_{0}^{b-a} u^{\operatorname{Re}(\beta)-1}\left|E_{\alpha, \beta, p}^{\gamma, \delta, q}\left(w u^{\alpha}\right)\right| d u=\beta
$$

then

$$
\begin{aligned}
\beta & =\sum_{n=0}^{\infty} \frac{\left|(\gamma)_{q n}\right|\left|w^{n}\right|}{\left|(\delta)_{p n}\right||\Gamma(\alpha n+\beta)|} \int_{0}^{b-a} u^{\operatorname{Re}(\alpha) n+\operatorname{Re}(\beta)-1} d u \\
& =(b-a)^{\operatorname{Re}(\beta)} \sum_{n=0}^{\infty} \frac{\left|(\gamma)_{q n}\right|\left|w(b-a)^{\operatorname{Re}(\alpha)}\right|^{n}}{\left|(\delta)_{p n}\right||\Gamma(\alpha n+\beta)||\operatorname{Re}(\alpha) n+\operatorname{Re}(\beta)|} .
\end{aligned}
$$

Hence,

$$
\left\|\mathscr{E}_{\alpha, \beta, p, w, \infty}^{\gamma, \delta} \varphi\right\|_{1} \leq \int_{a}^{\infty} \beta|\varphi(t)| d t \leq \beta\|\varphi\|_{1}
$$

We consider now composition of Weyl fractional integration and differentiation $I_{-}^{\lambda}, D_{-}^{\lambda}$ with the operator $\mathscr{E}_{\alpha, \beta, p, w, \infty}^{\gamma, \delta, q}$ defined in (9) contained in the next two theorems.

Remark 4. One can use the result of the next lemma for the proof of the stated theorems (see [5]).

Lemma 5. Let $a \in \mathbb{R}_{+}, \alpha, \beta, \gamma, \delta, \lambda \in \mathbb{C} ; \min \{\operatorname{Re}(\alpha), \operatorname{Re}(\beta)$, $\operatorname{Re}(\gamma), \operatorname{Re}(\delta), \operatorname{Re}(\lambda)\}>0$ and $p, q>0$, then for $x>a$, one has

$$
\begin{aligned}
& I_{a^{+}}^{\lambda}\left[(t-a)^{\beta-1} E_{\alpha, \beta, p}^{\gamma, \delta, q}\left[w(t-a)^{\alpha}\right]\right](x) \\
& \quad=(x-a)^{\beta+\lambda-1} E_{\alpha, \beta+\lambda, p}^{\gamma, \delta, q}\left[w(x-a)^{\alpha}\right] .
\end{aligned}
$$

Theorem 6. Let $\alpha, \beta, \gamma, \delta, \lambda, w \in \mathbb{C}$; with $\min \{\operatorname{Re}(\alpha), \operatorname{Re}(\beta)$, $\operatorname{Re}(\gamma), \operatorname{Re}(\delta)\}>0$ and $p, q>0$, then

$$
\begin{aligned}
\left(I_{-}^{\lambda} \mathscr{E}_{\alpha, \beta, p, w, \infty}^{\gamma, \delta, q} \varphi\right)(x) & =\left(\mathscr{E}_{\alpha, \beta+\lambda, p, w, \infty}^{\gamma, \delta, q} \varphi\right)(x) \\
& =\left(\mathscr{E}_{\alpha, \beta, p, w, \infty}^{\gamma, \delta, q} I_{-}^{\lambda} \varphi\right)(x)
\end{aligned}
$$

Proof. Applying (8) and (9), and by using Dirichlet formula (11) yields

$$
\begin{aligned}
& \left(I_{-}^{\lambda} \mathscr{E}_{\alpha, \beta, p, w, \infty}^{\gamma, \delta, q} \varphi\right)(x) \\
& =\frac{1}{\Gamma(\lambda)} \\
& \quad \times\left(\int_{x}^{\infty}(u-x)^{\lambda-1}\right. \\
& \left.\quad \times\left[\int_{u}^{\infty}(t-u)^{\beta-1} E_{\alpha, \beta, p}^{\gamma, \delta, q}\left[w(t-u)^{\alpha}\right] \varphi(t) d t\right]\right) d u \\
& =\int_{x}^{\infty} \frac{1}{\Gamma(\lambda)} \\
& \quad \times\left[\int_{x}^{t}(u-x)^{\lambda-1}(t-u)^{\beta-1} E_{\alpha, \beta, p}^{\gamma, \delta, q}\left[w(t-u)^{\alpha}\right] d u\right] \\
& \quad \times \varphi(t) d t .
\end{aligned}
$$

Let

$$
\tau=(t-u)
$$

then

$$
\begin{aligned}
& \left(I_{-}^{\lambda} \mathscr{E}_{\alpha, \beta, p, w, \infty}^{\gamma, \delta, q} \varphi\right)(x) \\
& \quad=\int_{x}^{\infty} \frac{1}{\Gamma(\lambda)}\left[\int_{0}^{t-x}(t-x-\tau)^{\lambda-1}\right.
\end{aligned}
$$

$$
\left.\times \tau^{\beta-1} E_{\alpha, \beta, p}^{\gamma, \delta, q}\left(w \tau^{\alpha}\right) d t\right] \varphi(t) d t .
$$

Applying (13) and the result of Lemma 5, we get

$$
\begin{aligned}
& \left(I_{-}^{\lambda} \mathscr{E}_{\alpha, \beta, p, w, \infty}^{\gamma, \delta, q} \varphi\right)(x) \\
& \quad=\int_{x}^{\infty} I_{0}^{\lambda}\left[\tau^{\beta-1} E_{\alpha, \beta, p}^{\gamma, \delta, q}\left(w \tau^{\alpha}\right)\right](t-x) \varphi(t) d t \\
& =\int_{x}^{\infty}(t-x)^{\beta+\lambda-1} E_{\alpha, \beta+\lambda, p}^{\gamma, \delta, q}\left[w(t-x)^{\alpha}\right] \varphi(t) d t \\
& =\left(\mathscr{E}_{\alpha, \beta+\lambda, p, w, \infty}^{\gamma, \delta, q} \varphi\right)(x) .
\end{aligned}
$$

On the other hand,

$$
\begin{aligned}
& \left(\mathscr{E}_{\alpha, \beta, p, w, \infty}^{\gamma, \delta, q} I_{-}^{\lambda} \varphi\right)(x) \\
& =\int_{x}^{\infty}(t-x)^{\beta-1} E_{\alpha, \beta, p}^{\gamma, \delta, q}\left[w(t-x)^{\alpha}\right] \frac{1}{\Gamma(\lambda)} \\
& \quad \times\left[\int_{t}^{\infty}(u-t)^{\lambda-1} \varphi(u) d u\right] d t \\
& =\int_{x}^{\infty} \frac{1}{\Gamma(\lambda)}\left[\int_{x}^{u}(t-x)^{\beta-1}(u-t)^{\lambda-1} E_{\alpha, \beta, p}^{\gamma, \delta, q}\left[w(t-x)^{\alpha} d t\right]\right] \\
& \quad \times \varphi(u) d u .
\end{aligned}
$$


Let $\tau=t-x$, we get

$$
\begin{aligned}
& \left(\mathscr{E}_{\alpha, \beta, p, w, \infty}^{\gamma, \delta, q} I_{-}^{\lambda} \varphi\right)(x) \\
& =\int_{x}^{\infty} \frac{1}{\Gamma(\lambda)}\left[\int_{0}^{u-x}(u-x-\tau)^{\lambda-1}\right. \\
& \left.\quad \times \tau^{\beta-1} E_{\alpha, \beta, p}^{\gamma, \delta, q}\left(w \tau^{\alpha}\right) d \tau\right] \varphi(u) d u .
\end{aligned}
$$

Returning to (13) and Lemma 5, we have

$$
\begin{aligned}
& \left(\mathscr{E}_{\alpha, \beta, p, w, \infty}^{\gamma, \delta, q} I_{-}^{\lambda} \varphi\right)(x) \\
& =\int_{x}^{\infty} I_{0}^{\lambda}\left[\tau^{\beta-1} E_{\alpha, \beta, p}^{\gamma, \delta, q}\left(w \tau^{\alpha}\right)\right](u-x) \varphi(u) d u \\
& =\int_{x}^{\infty}(u-x)^{\beta+\lambda-1} E_{\alpha, \beta+\lambda, p}^{\gamma, \delta, q}\left[w(u-x)^{\alpha}\right] \varphi(u) d u \\
& =\left(\mathscr{E}_{\alpha, \beta+\lambda, p, w, \infty}^{\gamma, \delta, q} \varphi\right)(x)
\end{aligned}
$$

which ends the proof.

A similar result concerning the Weyl fractional differentiation is stated in the following theorem.

Theorem 7. If the condition of Theorem 6 is satisfied, then

$$
\left(D_{-}^{\lambda} \mathscr{E}_{\alpha, \beta, p, w, \infty}^{\gamma, \delta, q} \varphi\right)(x)=\left(\mathscr{E}_{\alpha, \beta-\lambda, p, w, \infty}^{\gamma, \delta, q} \varphi\right)(x)
$$

Proof. Making use of (8), we get

$$
\left(D_{-}^{\lambda} \mathscr{E}_{\alpha, \beta, p, w, \infty}^{\gamma, \delta, q} \varphi\right)(x)=(-1)^{n}\left(\frac{d}{d x}\right)^{n}\left(I_{-}^{n-\lambda} \mathscr{E}_{\alpha, \beta, p, w, \infty}^{\gamma, \delta, q} \varphi\right)(x)
$$

and applying Theorem 6 yields

$$
\begin{aligned}
\left(D_{-}^{\lambda} \mathscr{E}_{\alpha, \beta, p, w, \infty}^{\gamma, \delta, q} \varphi\right)(x) \\
=(-1)^{n}\left(\frac{d}{d x}\right)^{n} \\
\quad \times \int_{x}^{\infty}(t-x)^{\beta+n-\lambda-1} E_{\alpha, \beta-n-\lambda, p}^{\gamma, \delta, q}\left[w(t-x)^{\alpha}\right] \varphi(t) d t .
\end{aligned}
$$

By using Dirichlet formula (12), we get

$$
\begin{gathered}
\left(D_{-}^{\lambda} \mathscr{E}_{\alpha, \beta, p, w, \infty}^{\gamma, \delta, q} \varphi\right)(x) \\
=(-1)^{n}\left(\frac{d}{d x}\right)^{n-1}
\end{gathered}
$$

$$
\begin{aligned}
& \times\left(\int_{x}^{\infty} \frac{\partial}{\partial x}(t-x)^{\beta+n-\lambda-1} E_{\alpha, \beta+n-\lambda, p}^{\gamma, \delta, q}\left[w(t-x)^{\alpha}\right] \varphi(t) d t\right) \\
&+ \lim _{t \rightarrow x^{+}}(t-x)^{\beta+n-\lambda-1} E_{\alpha, \beta+n-\lambda, p}^{\gamma, \delta, q}\left[w(t-x)^{\alpha}\right] \varphi(t) \\
&=(-1)^{n}\left(\frac{d}{d x}\right)^{n-1} \\
& \times \int_{x}^{\infty} \sum_{n=0}^{\infty} \frac{(\gamma)_{q n} w^{n}(\alpha n+\beta+n-\lambda-1)}{(\delta)_{p n} \Gamma(\alpha n+\beta-n-\lambda)} \\
& \times(-1)^{n}\left(\frac{d}{d x}\right)^{n-1} \int_{x}^{\infty}(-1)(t-x)^{\beta+n-\lambda-2} \\
& \times E_{\alpha, \beta+n-\lambda-1, p}^{\gamma, \delta, q}\left[w(t-x)^{\alpha}\right] \cdot \varphi(t) d t .
\end{aligned}
$$

Repeating this process $n-1$ times, we get

$$
\begin{aligned}
& \left(D_{-}^{\lambda} \mathscr{E}_{\alpha, \beta, p, w, \infty}^{\gamma, \delta, q} \varphi\right)(x) \\
& =(-1)^{n}(-1)^{n} \int_{x}^{\infty}(t-x)^{\beta-\lambda-1} E_{\alpha, \beta-\lambda, p}^{\gamma, \delta, q}\left[w(t-x)^{\alpha}\right] \varphi(t) d t \\
& =\left(\mathscr{E}_{\alpha, \beta-\lambda, p, w, \infty}^{\gamma, \delta, q} \varphi\right)(x) .
\end{aligned}
$$

\section{References}

[1] G. M. Mittag-Leffler, "Sur la nouvelle function $E_{\alpha}(z)$," Comptes Rendus de l'Académie des Sciences, vol. 137, pp. 554-558, 1903.

[2] A. Wiman, "Über den Fundamentalsatz in der Teorie der Funktionen $E_{\alpha}(x)$," Acta Mathematica, vol. 29, no. 1, pp. 191-201, 1905.

[3] T. R. Prabhakar, "A singular integral equation with a generalized Mittag-Leffler function in the kernel," Yokohama Mathematical Journal, vol. 19, pp. 7-15, 1971.

[4] A. K. Shukla and J. C. Prajapati, "On a generalization of MittagLeffler function and its properties," Journal of Mathematical Analysis and Applications, vol. 336, no. 2, pp. 797-811, 2007.

[5] T. O. Salim and A. W. Faraj, "A generalization of MittagLeffler function and integral operator associated with fractional calculus," Journal of Fractional Calculus and Applications, vol. 3, no. 5, pp. 1-13, 2012.

[6] A. A. Kilbas, M. Saigo, and R. K. Saxena, "Generalized MittagLeffler function and generalized fractional calculus operators," Integral Transforms and Special Functions, vol. 15, no. 1, pp. 3149, 2004.

[7] H. M. Srivastava and Z. Tomovski, "Fractional calculus with an integral operator containing a generalized Mittag-Leffler function in the kernel," Applied Mathematics and Computation, vol. 211, no. 1, pp. 198-210, 2009.

[8] S. G. Samko, A. A. Kilbas, and O. I. Marichev, Fractional Integrals and Derivatives. Theory and Applications, Gordon and Breach Science Publishers, Yverdon, Switzerland, 1993.

[9] I. N. Sneddon, The Use of Integral Transforms, Tata McGraw Hill, New Delhi, India, 1979. 


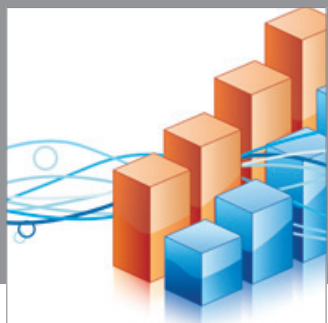

Advances in

Operations Research

mansans

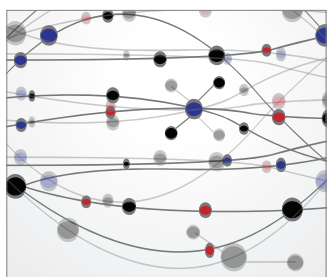

The Scientific World Journal
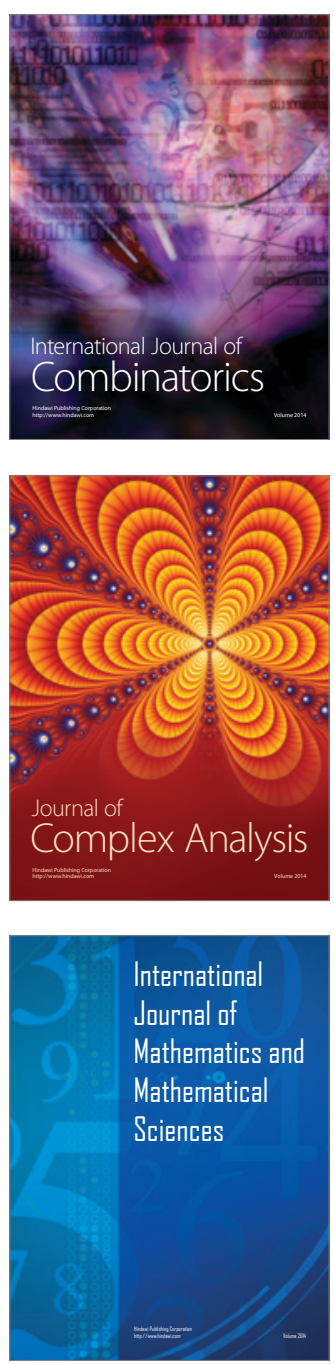
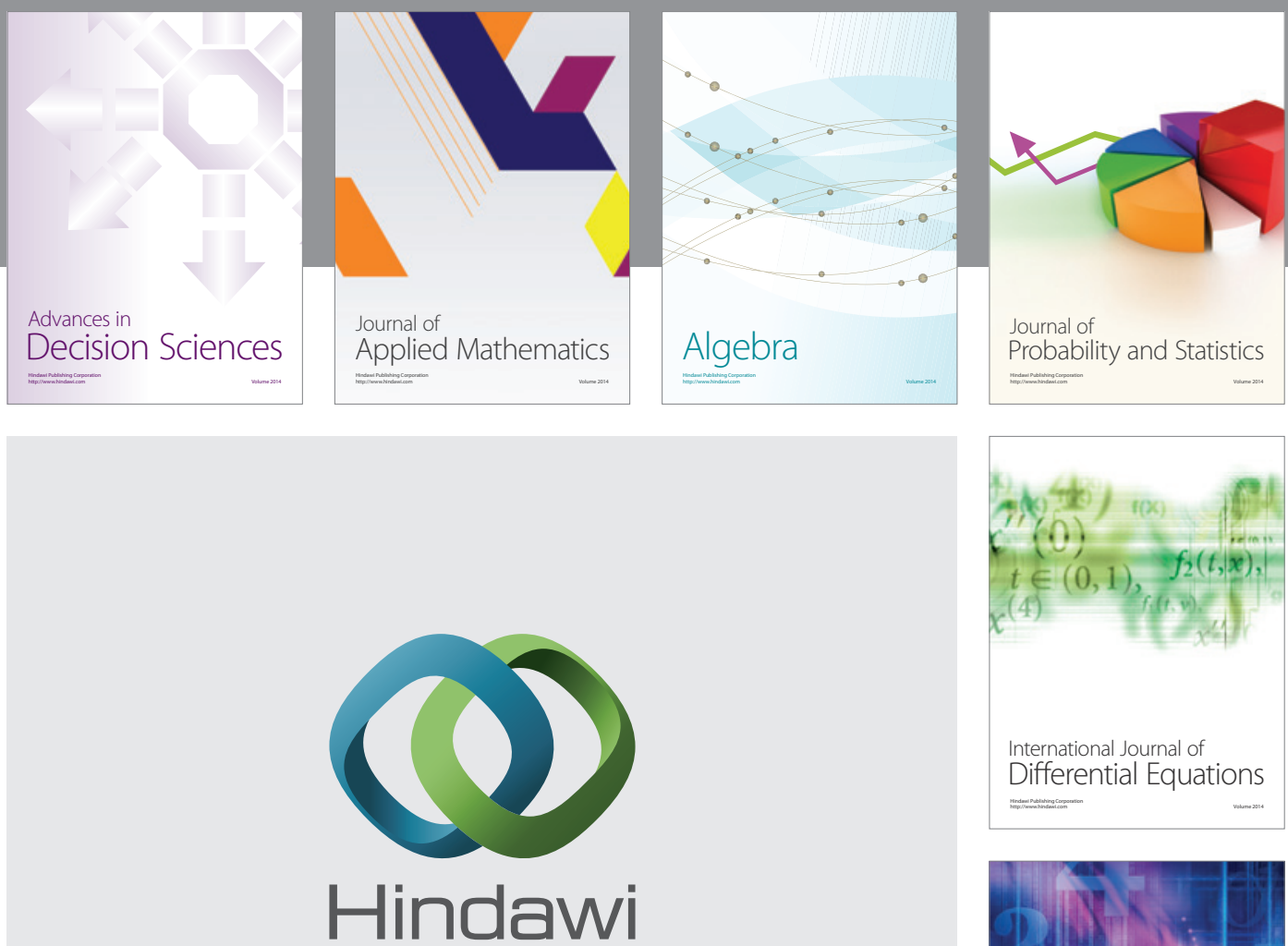

Submit your manuscripts at http://www.hindawi.com
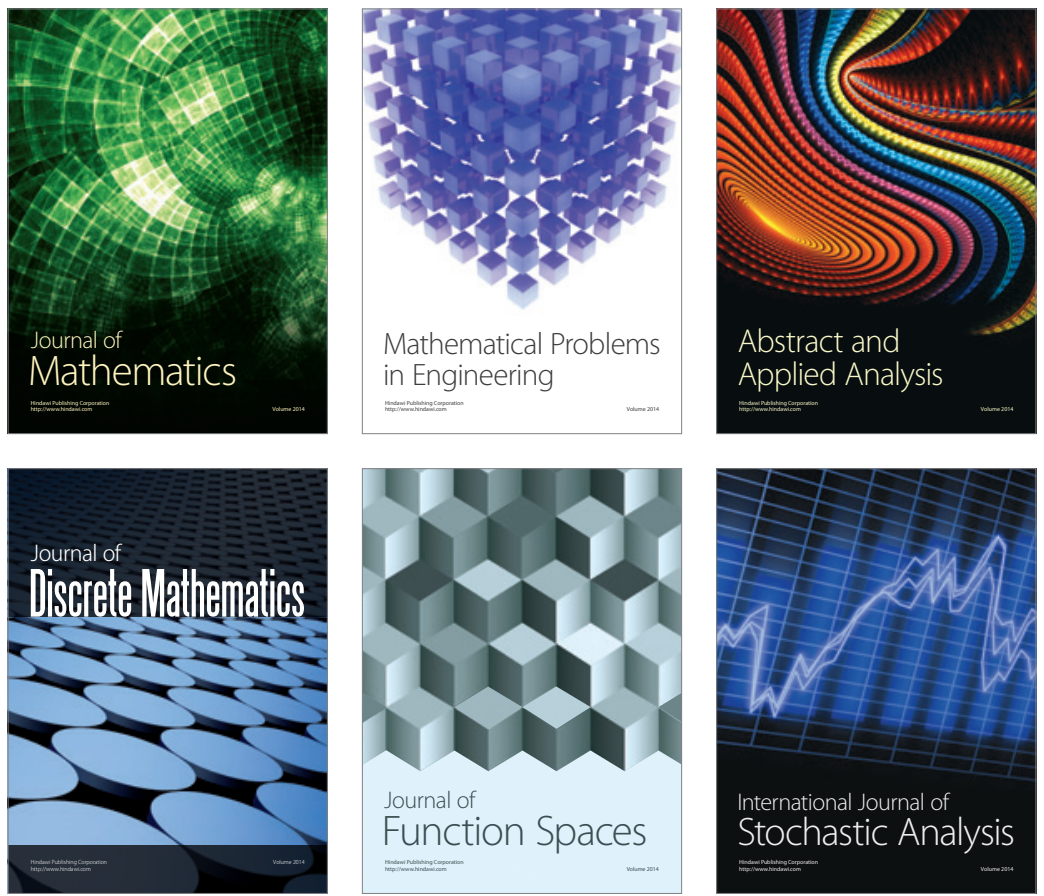

Journal of

Function Spaces

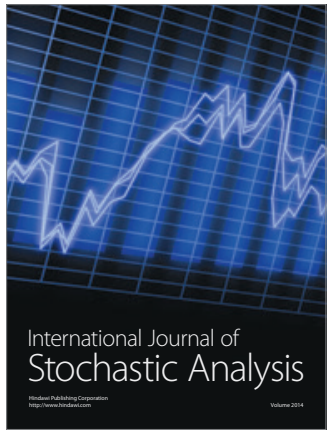

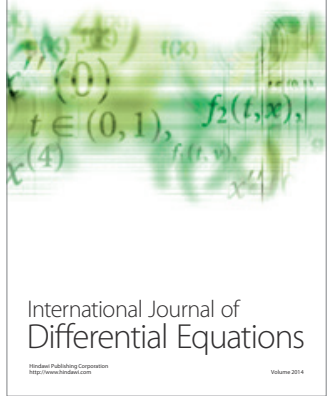
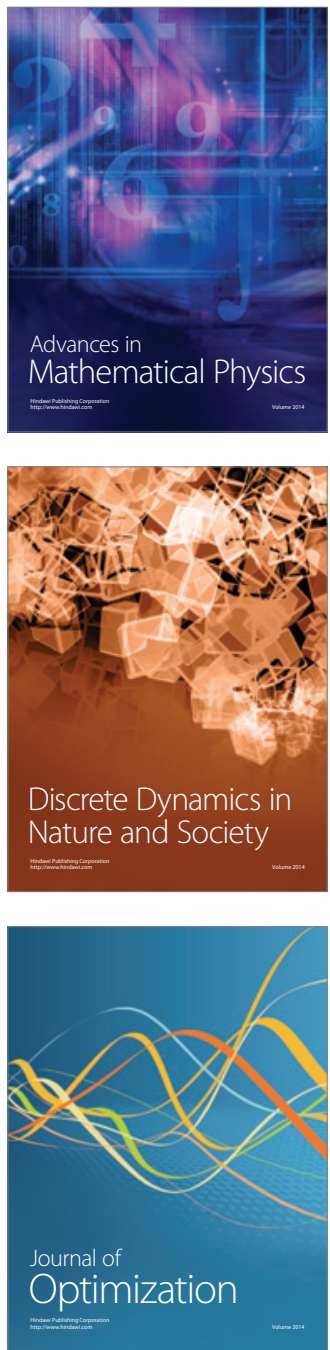